\title{
USO DE REDES NEURAIS ARTIFICIAIS NA IDENTIFICAÇÃO DE VOCALIZAÇÃO DE SUÍNOS
}

\section{IRENILZA DE A. NÄÄS ${ }^{1}$, LEONARDO S. L. CAMPOS ${ }^{2}$, MARTA DOS S. BARACHO ${ }^{3}$, YAMILIA B. TOLON ${ }^{4}$}

\begin{abstract}
RESUMO: O bem-estar dos animais tem sido importante tópico de pesquisa na produção animal, principalmente no tocante às formas de sua avaliação. Na avaliação do bem-estar animal, a vocalização mostra-se como ferramenta interessante, por fornecer dados de forma não-invasiva, podendo também ser facilmente automatizada. O presente trabalho teve o objetivo de implementar algoritmo baseado em redes neurais artificiais, capaz de reconhecer vocalizações relacionadas com padrões indicativos de bem-estar. A pesquisa teve duas partes, sendo a primeira o desenvolvimento do algoritmo, e a segunda, sua validação com dados de campo. Registros prévios permitiram o desenvolvimento do algoritmo, a partir de comportamentos observados em porcas alojadas em gaiolas de maternidade. O software Matlab $^{\circledR}$ foi utilizado na implementação da rede. Foi selecionado um algoritmo de gradiente de retropropagação para treinar a rede com os seguintes critérios de parada: máximo de 5.000 iterações ou soma quadrática do erro menor que 0,1 . A validação deu-se com porcas e leitões alojados em granja comercial. Dentre os comportamentos usuais, os que mereceram destaque foram: a disputa por alimento no momento das mamadas e o eventual risco de agressão involuntária entre os leitões ou entre esses e a porca. O algoritmo foi capaz de reconhecer, por meio da intensidade do ruído, a situação inerente ao risco de redução do bem-estar dos leitões.
\end{abstract}

PALAVRAS-CHAVE: bem-estar animal, leitões, análise de sinais.

\section{THE USE OF ARTIFICIAL NEURAL NETWORK IN IDENTIFYING SWINE VOCALIZATION}

\begin{abstract}
Animal welfare has been an important research topic in animal production mainly in its ways of assessment. Vocalization is found to be an interesting tool for evaluating welfare as it provides data in a non-invasive way as well as it allows easy automation of process. The present research had as objective the implementation of an algorithm based on artificial neural network that had the potential of identifying vocalization related to welfare pattern indicatives. The research was done in two parts, the first was the development of the algorithm, and the second its validation with data from the field. Previous records allowed the development of the algorithm from behaviors observed in sows housed in farrowing cages. Matlab ${ }^{\circledR}$ software was used for implementing the network. It was selected a retropropagation gradient algorithm for training the network with the following stop criteria: maximum of 5,000 interactions or error quadratic addition smaller than 0.1 . Validation was done with sows and piglets housed in commercial farm. Among the usual behaviors the ones that deserved enhancement were: the feed dispute at farrowing and the eventual risk of involuntary aggression between the piglets or between those and the sow. The algorithm was able to identify through the noise intensity the inherent risk situation of piglets' welfare reduction.
\end{abstract}

KEYWORDS: animal welfare, piglets, signal analysis.

\footnotetext{
${ }^{1}$ Eng $^{\circ}$ Civil, Professora Colaboradora, FEAGRI-UNICAMP, Campinas - SP, irenilza@agr.unicamp.br

${ }^{2}$ Eng $^{\mathrm{o}}$ Mecatrônico, FEM, UNICAMP, Campinas - SP.

${ }^{3}$ Bióloga, Pesquisadora Colaboradora, FEAGRI-UNICAMP, Campinas - SP.

${ }^{4}$ Zootecnista, Pesquisadora Colaboradora, FEAGRI-UNICAMP, Campinas - SP.

Recebido pelo Conselho Editorial em: 20-8-2007

Aprovado pelo Conselho Editorial em: 3-6-2008
} 


\section{INTRODUÇÃO}

A organização do mercado internacional de carne trouxe à cena, nos últimos anos, a questão do bem-estar animal. Segundo vários autores (ROLLING, 1995; GRANDIN, 1998; HEIER et al., 2002; DAWKINS, 2003), a necessidade de aumento da produção, com significativa redução de espaço, levou à produção mais intensificada, em que os animais, com menos liberdade de resposta e em um ambiente mais agressivo, ficaram mais propícios a doenças e ao sofrimento. Além de se conhecer condições de bem-estar, indicadas em função da espécie e de sua produtividade (ALGERS \& JENSEN, 1985; FRASER \& BROOM, 1990; WENNRICH, 1981; PRESCOTT \& WATHES, 2002; Le NEINDRE et al., 2004; McINERNEY, 2004), é necessário que se dispusesse de formas práticas para mensurá-la.

Dentre as formas de avaliação do bem-estar animal, a vocalização apresenta-se como importante ferramenta (WEARY et al., 1997; MANTEUFFEL \& SCHÖN, 2002; HAY et al., 2003; MANTEUFFEL et al., 2004), utilizando sistemas de monitoramento não-invasivos, que podem ser, inclusive, automatizados (FRASER, 1974). Animais utilizam a vocalização como forma de comunicação entre indivíduos da mesma espécie (GRANDIN, 1998), podendo ocorrer de forma espontânea ou ser resultado de um evento externo que, por serem emocionalmente relevantes, são capazes de estimular uma complexa rede do sistema nervoso central (MANTEUFFEL \& SCHÖN, 2002).

A vocalização na produção de suínos tem motivado o interesse de cientistas (JENSEN \& ALGERS, 1984; WEARY et al., 1997; SCHÖN et al., 1999; WEARY et al., 1998; BECKER, 2004), principalmente na maternidade, pois nela a comunicação porca-leitão é indicativo das necessidades dos leitões. ALGERS \& JENSEN (1985) analisaram a comunicação de suínos durante a amamentação, encontrando padrões de vocalização distintos, que estiveram significantemente relacionados com situações específicas, como: roncos, grito, grunhido leve e profundo, além de pareceram afetar o comportamento da reprodutora. WEARY et al. (1997) encontraram que os leitões isolados aumentaram o número de vocalizações quando expostos a gravações de chamadas da porca, ou ainda quando o ambiente estava fora do ideal, concluindo que leitões manifestam suas reações por meio da vocalização.

Técnicas para extrair características da vocalização, como extração no domínio do tempo e no da freqüência, podem utilizar o modelo Cesptrum (no qual as periódicas são separadas das nãoperiódicas) e codificação de predição linear. Todas essas características são analisadas por várias metodologias, como análises estatísticas, modelos ocultos de Markov e as Redes Neurais Artificiais. Essas redes têm como fonte de inspiração a analogia neurobiológica em que o elemento básico, o neurônio, encontra-se em grande quantidade, formando módulos complexos maciçamente conectados entre si para a realização de determinadas tarefas (HAYKIN, 2001).

MANTEUFFEL \& SCHÖN (2002) criaram um sistema que, por meio dos coeficientes de predição linear, aliados a uma rede neural artificial, foi capaz de reconhecer vocalizações relacionadas ao estresse em ambiente com ruído com pequeno erro de reconhecimento $(<5 \%)$. CHEDAD et al. (2001) implementaram um algoritmo baseado em redes neurais probabilísticas para detectar tosse em suínos, diferenciando-a entre outros ruídos de fundo, alcançando altos níveis de detecção (91,9\%). REBY et al. (1997) propuseram metodologia baseada em redes neurais, utilizando o gradiente de retropropagação, obtendo bons resultados no reconhecimento de indivíduos de um grupo de quatro cervos.

O objetivo desta pesquisa foi criar um algoritmo baseado em redes neurais artificiais, capaz de reconhecer e classificar vocalizações relacionadas com determinados comportamentos existentes na maternidade de suínos.

\section{MATERIAL E MÉTODOS}

O experimento consistiu de duas etapas: primeiro foi definido um algoritmo de aprendizagem adequado ao problema, feito o treinamento da rede neural por meio da apresentação do conjunto de 
exemplos gravados durante a coleta de dados, junto com a resposta desejada à rede já estruturada e, em seguida, a validação do algoritmo em granja comercial.

\section{Desenvolvimento do algoritmo}

Foi proposta uma arquitetura do sistema de auxílio (Figura 1), em que o sistema era composto por três subsistemas:

1 - Observador: alimentado com os dados gravados do experimento, em que o usuário, a partir da sua observação, salva os momentos nos quais ocorre o comportamento de estudo; o programa solicita, então, um rótulo para o conhecimento do comportamento gravado.

2 - Analisador: alimentado com os dados do Observador, realiza os cálculos dos espectros definidos pelo usuário, possibilita a visualização e também gera o pacote dos dados calculados juntamente com seus rótulos.

3 - Rede Neural: alimentada com os dados do Analisador, realiza o treinamento da rede e permite a validação da rede.

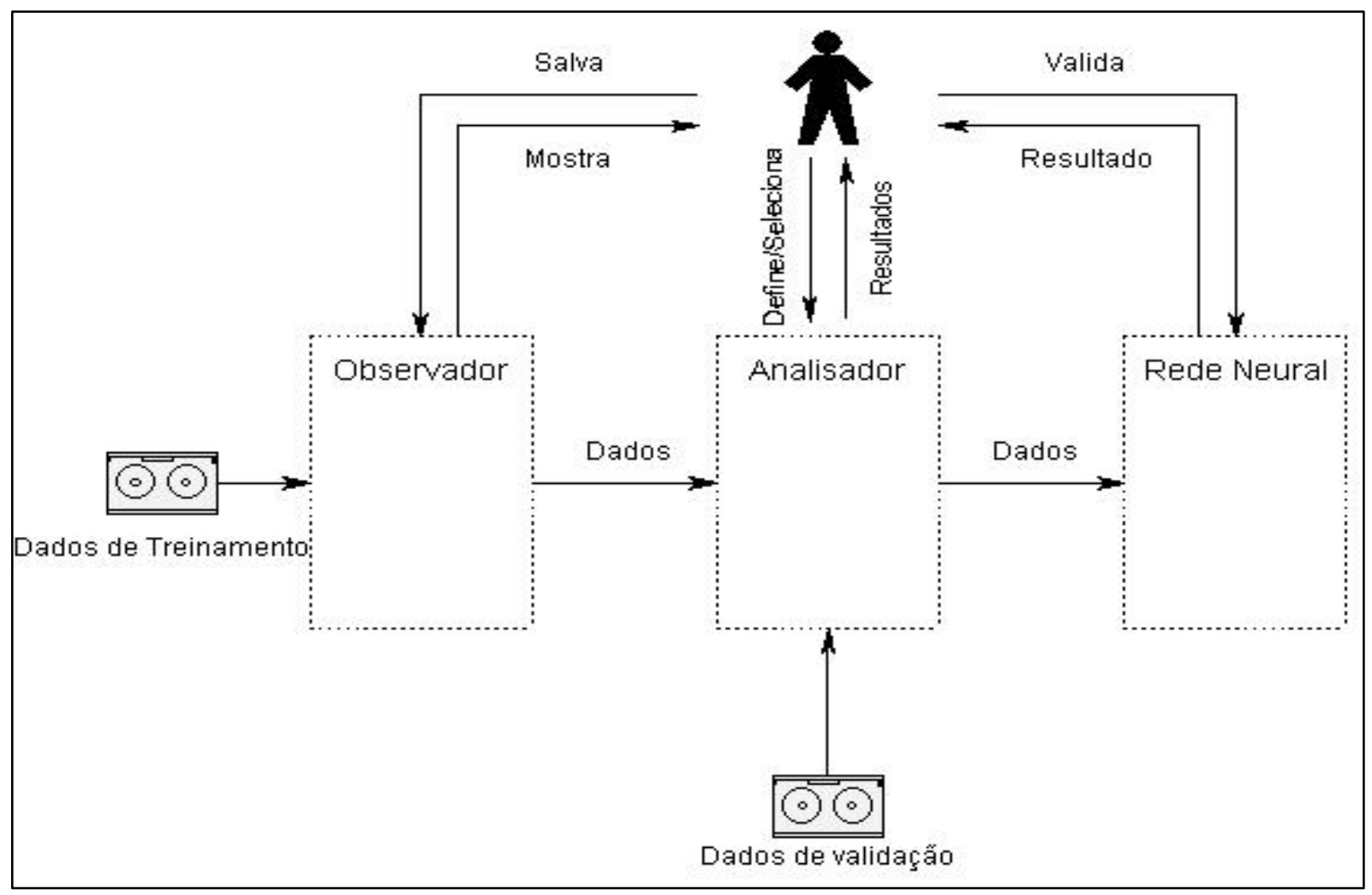

FIGURA 1. Esquema da arquitetura do sistema de auxílio ao reconhecimento de estresse na maternidade de suínos. Layout of the system architecture for helping recognizing stress in swine nursing.

Para o treinamento da rede, foram selecionadas, de forma aleatória, duas reprodutoras em baias individuais (R1 e R2) e uma reprodutora em baia coletiva (R3), com um microfone Clone 11013 conectado a um computador Móbile Intel ${ }^{\circledR}$, processador Pentium $^{\circledR} 4,3,33 \mathrm{GHz}$ e memória de $512 \mathrm{MB}$, tendo sido calculadas as formas de ondas dos sons emitidos pela porca durante a amamentação e seu respectivo espectro de freqüência. Para a análise do comportamento dos suínos, foi utilizada uma câmera digital (JVC GDR-120U - 30 Hz, com 520 linhas de resolução horizontal).

Foi escolhido o algoritmo de gradiente de retropropagação para treinar a rede com os seguintes critérios de parada: máximo de 5.000 iterações ou soma quadrática do erro menor que 0.1.

Com a utilização do software Audacity ${ }^{\circledR}$, Versão $1.2 .4 \mathrm{~b}$, os sons foram analisados pelos seus espectros de freqüência e diagramas. Os dados gravados compuseram o conjunto de exemplos para 
o aprendizado da RNA (Rede Neural Artificial), que auxiliou na proposição de modelo e de estrutura adequada de RNA.

\section{Validação do algoritmo}

A validação do experimento foi realizada em granja comercial de produção de suínos, no Estado de São Paulo, situada em latitude $23^{\circ} 12^{\prime} 10^{\prime}$ ' S, longitude $47^{\circ} 17^{\prime} 35^{\prime}$ ' W, e altitude média de $521 \mathrm{~m}$, em sala de maternidade.

Foi utilizada uma câmera de gravação em $8 \mathrm{~mm}$ da marca JVC, e foram gravados imagens e sons simultaneamente. Foram escolhidas ao acaso sete baias com gaiolas de maternidade, e cada baia proveu amostras de 5 a 10 minutos. Durante a coleta, primeiramente, procurou-se direcionar a câmera para o local onde havia o maior número de leitões e, uma vez posicionada a câmera, essa era mantida na mesma posição durante a amostragem. Posteriormente, os dados foram digitalizados em formato DVD e, por meio da visualização das imagens, foram gerados relatórios de comportamentos observados. $\mathrm{O}$ estudo baseou-se em comportamentos acompanhados de vocalizações, sendo o conteúdo de áudio dessas vocalizações gravado em formato WAV, com o auxílio do software Audacity ${ }^{\circledR}$, com taxa de amostragem de $44.100 \mathrm{~Hz}$ e resolução de 16 bits.

A partir desses dados gravados, foram extraídas as seguintes características no software MatLab $^{\circledR}$ : o espectro das freqüências, por meio da transformada de Fourier; o espectrograma, que mostra a distribuição espectral ao longo do tempo; a autocorrelação, e a intensidade sonora (“Loudness"), que representa a intensidade do som segundo a percepção humana.

Foi utilizada a ferramenta do MatLab ${ }^{\circledR}$ de Redes Neurais Artificiais e, após estruturada, a rede neural foi treinada de forma a ter a melhor eficiência possível, em que os parâmetros de entrada são passados pelo modelo criado. A rede implementada foi do tipo feed-forward, em que os pesos sinápticos da primeira camada vêm das entradas, e os pesos das camadas subseqüentes vêm das camadas anteriores. A função sigmóide logarítmica foi adotada como função de ativação. $O$ treinamento adotado foi baseado no algoritmo do gradiente de retropropagação, em que os pesos são alterados na direção que minimiza a função de performance (soma quadrática dos erros). A rede foi estruturada com três camadas; o número de neurônios da camada de entrada corresponde ao número de pontos que a entrada possui, ou seja, o número de pontos das abscissas dos respectivos gráficos: espectro de freqüência, intensidade sonora e autocorrelação. A segunda camada possui dez neurônios, e a terceira e última possui três, número suficiente para a quantidade de comportamentos encontrados.

\section{RESULTADOS E DISCUSSÃO}

\section{Análise dos sinais}

A partir dos dados gravados com as três reprodutoras (R1, R2 e R3), foram calculadas as formas de ondas dos sons emitidos pela porca durante a amamentação e seu respectivo espectro de freqüência de uma única vocalização (limitada por zeros na amplitude pela esquerda e direita da forma de onda), conforme Figuras 2; 3 e 4.

Nota-se diferença entre os formatos das ondas e o espectro do som emitido pelas reprodutoras, o que demonstra que a expressão de cada porca foi diferente frente a um mesmo tipo de evento, concordando com o que preconiza MANTEUFFEL et al. (2004) sobre a individualidade das respostas dos suínos.

A imagem foi analisada juntamente com um algoritmo que extrai da imagem o conteúdo de fundo (estimado pela mediana acumulada) e segue a movimentação dos indivíduos com um retângulo ao seu redor (por meio de um filtro Kalman), mostrada na Figura 5. 


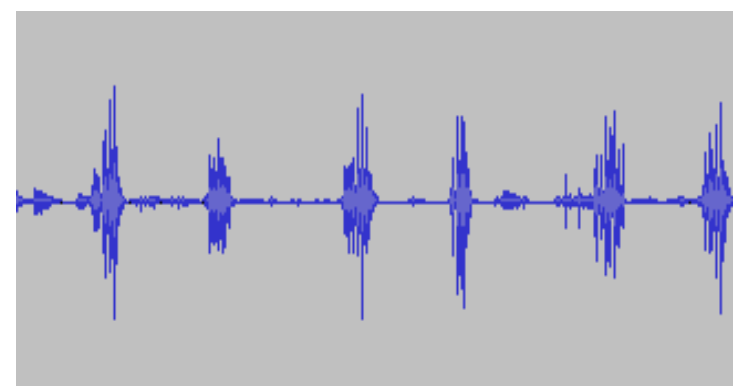

(a)

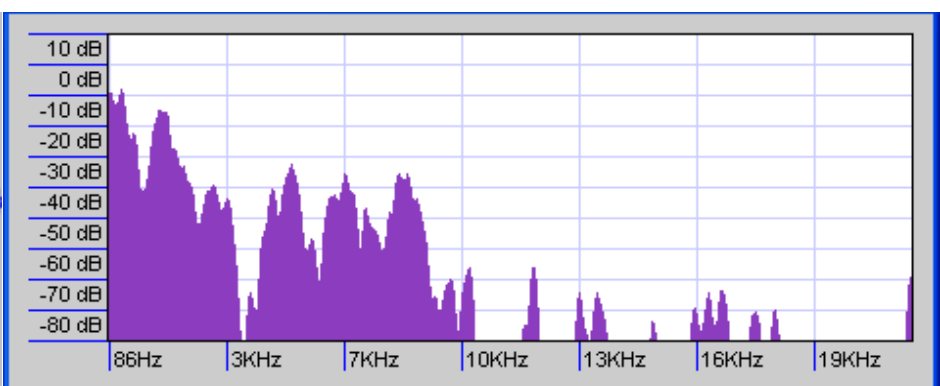

(b)

FIGURA 2. Formato de onda (a) e espectro (b) do som proveniente da reprodutora R1. Wave shape (a) and spectrum (b) of the noise from sow R1.

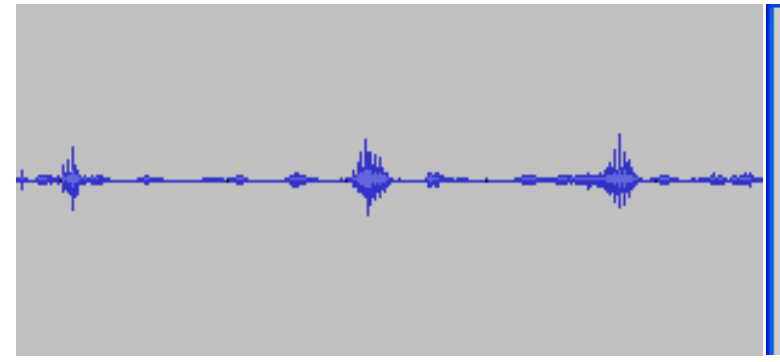

(a)

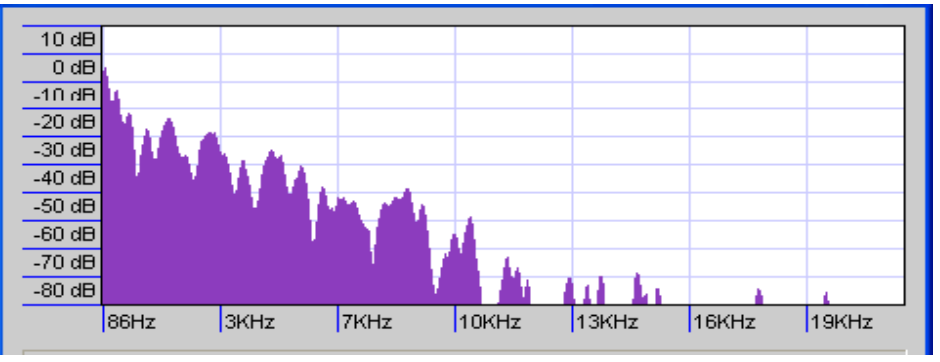

(b)

FIGURA 3. Formato de onda (a) e espectro (b) do som proveniente da reprodutora R2. Wave shape (a) and spectrum (b) of the noise from sow $R 2$.

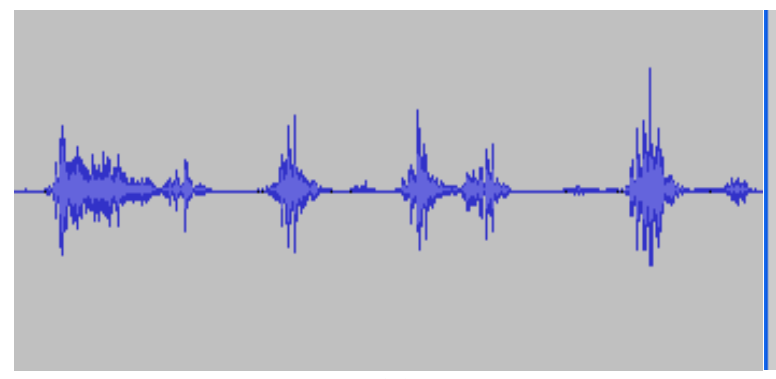

(a)

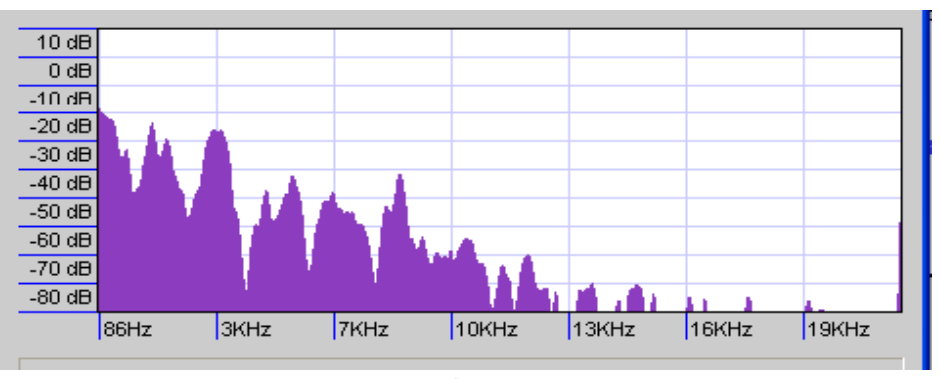

(b)

FIGURA 4. Formato de onda (a) e espectro (b) do som proveniente da reprodutora R3. Wave shape (a) and spectrum (b) of the noise from sow $R 3$.

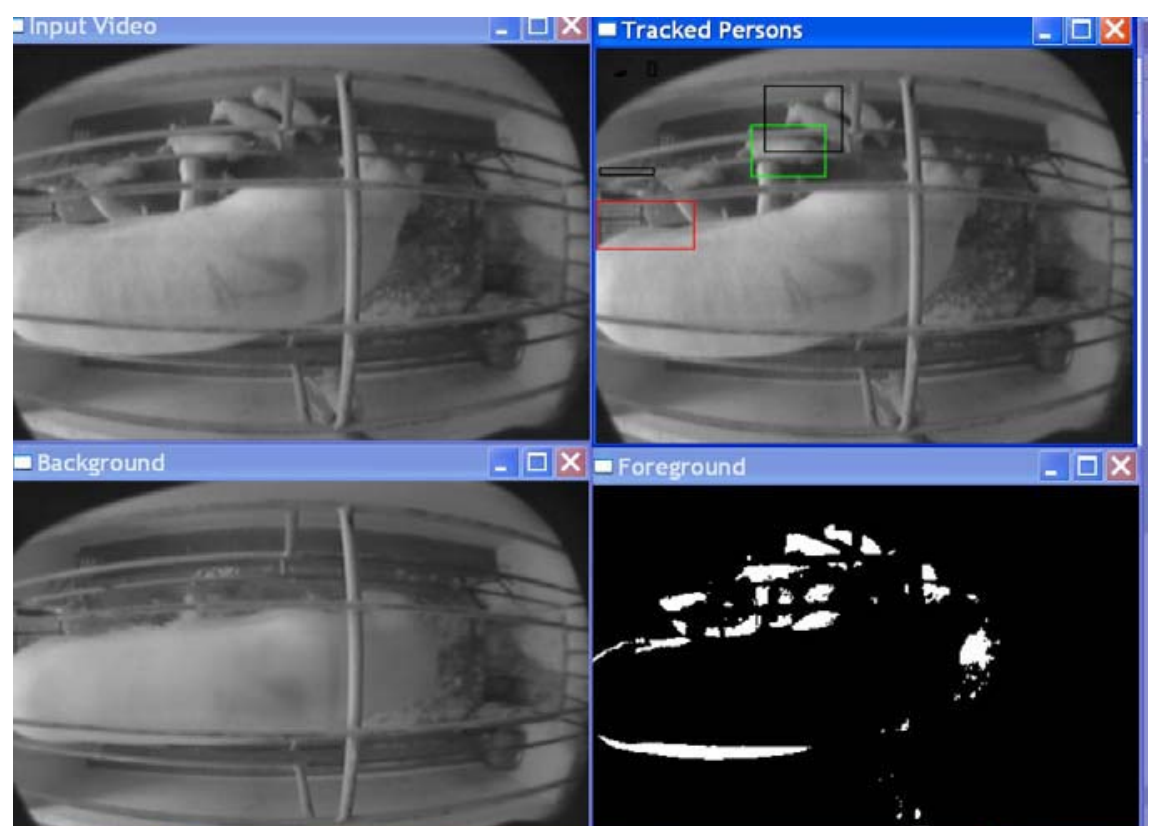

FIGURA 5. Registro da imagem da porca com a leitegada na gaiola na fase pré-desmame. Recording of the sow's image with the piglets in the cage during the pre-weaning phase. 
Segundo FRASER \& BROOM (1990), os experimentos que envolvem observações comportamentais devem ser cautelosamente planejados, para a obtenção de conclusões confiáveis. Uma precaução necessária é o efeito da presença de um observador no comportamento dos animais, o que foi previsto na rede, sendo a informação a respeito de um determinado comportamento obtida por vários métodos, incluindo o período de observação.

A definição dos parâmetros de entrada da rede baseou-se no custo computacional envolvido, portanto não foram consideradas as características no domínio do tempo, já que, neste caso, seria necessário que a rede se comportasse dinamicamente, demandando grandes quantidades de amostras e tempo de cálculo; fez-se, então, comparação para as seguintes entradas: espectro de freqüência (calculado em 512 pontos), autocorrelação (calculada em 2.049 pontos) e intensidade sonora (calculada em 240 pontos).

Foi possível identificar, na análise das imagens, quatro comportamentos distintos (Tabela 1), com diferentes freqüências (Figura 6), que possuíam vocalização associada, sendo que o comportamento $\mathrm{C} 4$ teve freqüência de ocorrência baixa e não foi considerado na análise.

TABELA 1. Etograma dos comportamentos observados e estudados. Ethogram of the observed and studied behaviors.

\begin{tabular}{cl}
\hline Comportamento & \multicolumn{1}{c}{ Definição } \\
\hline C1 & Disputa pela mama da porca entre os leitões \\
C2 & Busca dos leitões pela mama da porca \\
C3 & Eventos de autodefesa dos leitões quando sob a presença de algum risco \\
C4 & Interação entre os leitões por meio de massagens com focinho \\
\hline
\end{tabular}

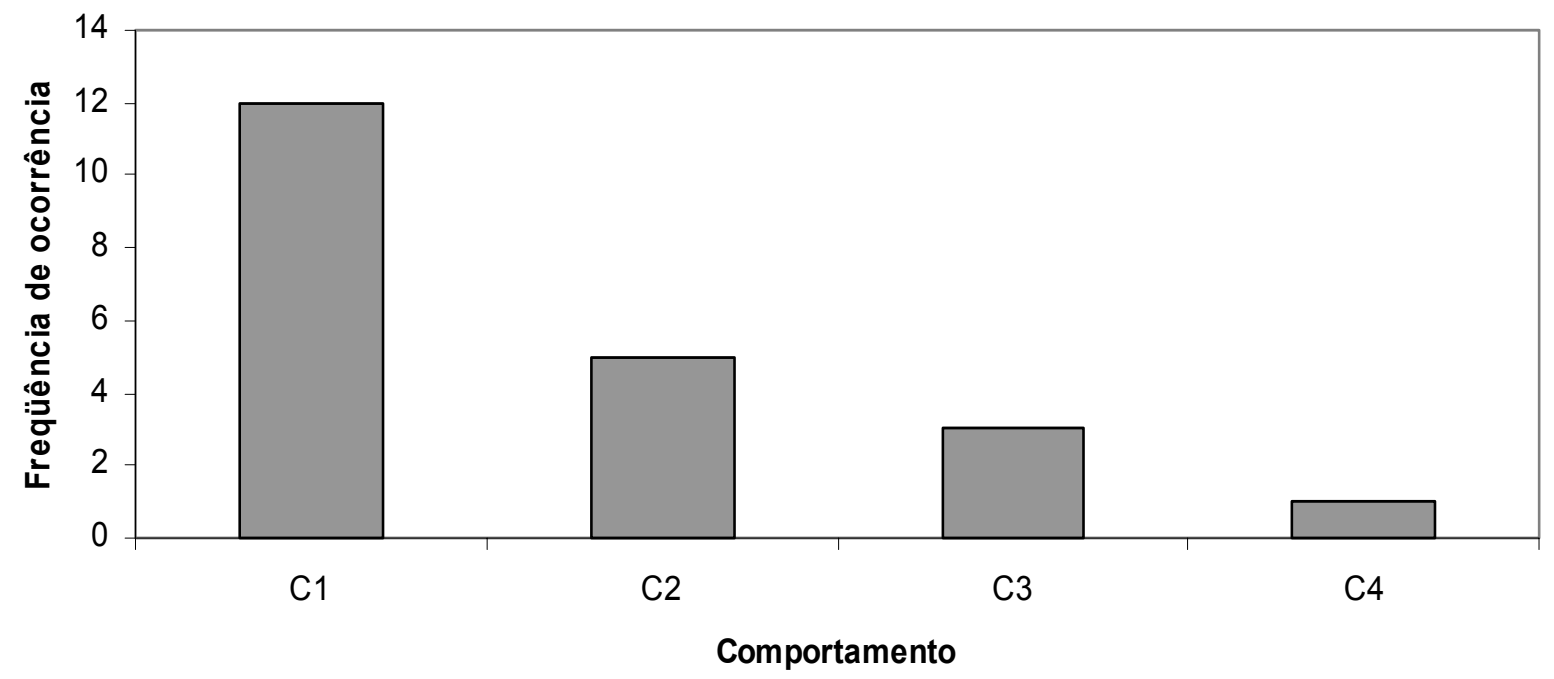

FIGURA 6. Distribuição dos comportamentos observados das porcas e leitões. Distribution of the sows and piglets observed behaviors.

A vocalização $\mathrm{C} 1$ ocorreu com maior freqüência e notou-se que a mesma aconteceu devido ao esforço que os leitões faziam para alcançar a mama da porca, uma vez que o espaço é reduzido pela presença dos outros indivíduos. A vocalização C2 foi direcionada à porca e foi possível notar sua ocorrência quando havia a massagem das mamas pelos leitões. Já a vocalização durante o comportamento $\mathrm{C} 3$ ocorreu quando o leitão esteve sob algum risco, por exemplo, quando as patas da porca os atingiam. A vocalização relacionada ao comportamento $\mathrm{C} 4$ foi resultante dos movimentos de interação entre leitões e, por apresentar baixa freqüência, não foi considerado na rede neural (Tabela 2).

A interpretação dos comportamentos foi difícil e, algumas vezes, não se identificou o emissor, não sendo possível correlacionar o comportamento à vocalização. Outras vezes, a vocalização 
proveniente de outras baias interferia na coleta de dados, diminuindo o número de amostras. Para sanar esse problema, foi necessário usar microfones diretivos e lançar mão de filtragem digital.

Um ponto interessante encontrado é que os leitões mais novos vocalizaram menos que os mais velhos; além disso, foram encontrados poucos padrões comportamentais associados com vocalização, diferente do que encontrou GRAUVOGL (1958), que distinguiu 23 tipos diferentes de expressões vocais relacionadas com distintos comportamentos. Entretanto, os poucos aqui identificados foram importantes na avaliação do bem-estar dos leitões, pois estavam relacionados com disputa por alimento, concordando com BECKER (2004).

\section{Desenvolvimento do algoritmo}

Como estrutura, servindo de ponto de partida, foi utilizada uma rede do tipo alimentada positivamente (feed-foward) com duas camadas, uma camada escondida de dez neurônios e a camada de saída com três neurônios. Na Figura 7, ilustra-se o esquema da rede para a intensidade sonora como entrada; na Tabela 2, apresenta-se a codificação da saída adotada.

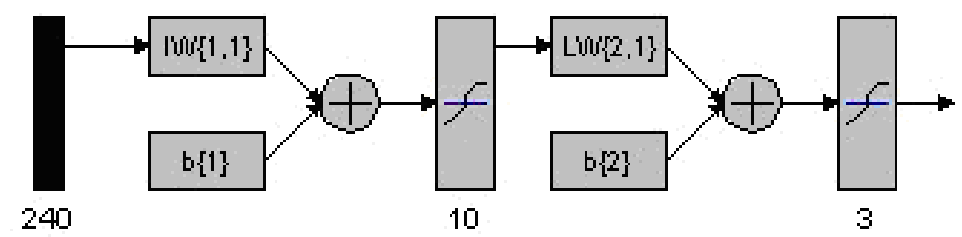

FIGURA 7. Esquema da rede feed-foward utilizada. Layout of the feed-forward network used.

TABELA 2. Codificação adotada para a rede a partir dos comportamentos apresentados pelos leitões e pela porca. Adopted code for the network from the behaviors presented by the sows and piglets.

\begin{tabular}{cc}
\hline Comportamento & Saída \\
\hline C1 & {$\left[\begin{array}{lll}0 & 0 & 1\end{array}\right]$} \\
C2 & {$\left[\begin{array}{lll}1 & 0 & 0\end{array}\right]$} \\
C3 & {$\left[\begin{array}{lll}0 & 1 & 0\end{array}\right]$} \\
\hline
\end{tabular}

As análises típicas encontradas em cada um dos quatro comportamentos são mostradas nas Figuras 8 a 10.

A avaliação do comportamento foi baseada nos fundamentos de FRASER \& BROOM (1990) e HAY et al. (2003). Segundo os autores, na seleção de formas de medições para um estudo particular, é útil conhecer o conjunto de comportamentos que um animal pode mostrar de forma detalhada e descritiva, isto é, formalizado no conjunto das observações (Tabela 1).

Vocalizações com baixa tonalidade (grunhidos) são utilizadas na manutenção do contato social, enquanto a alta tonalidade (gritos) está mais relacionada com estados de excitação (KILEY, 1972; FRASER, 1974). Pelos resultados mostrados na Figura 8, nota-se que o comportamento C1 registrou, no gráfico de dados normalizados, manutenção mais constante da tonalidade alta, o que, segundo SCHÖN et al. (1999), indica a disputa por alimento.

As expressões vocais de suínos podem ser contínuas ou descontínuas (KLINGHOLZ \& MEYNHARDT, 1979; JENSEN \& ALGERS, 1984), sendo altamente correlacionada a seus níveis de excitação. Nas Figuras 9 e 10, apresentam-se a intensidade sonora, a autocorrelação, o espectro de freqüências e o espectrograma para o ruído de uma amostra dos comportamentos C2 e C3, respectivamente. Pode-se notar, no gráfico de dados normalizados, que a busca das tetas da matriz pelos leitões (C2) apresenta uniformidade de baixa tonalidade, indicando situação de normalidade, o que concorda com os dados encontrados por MANTEUFFEL \& SCHÖN (2002). Já a variação da distribuição encontrada no comportamento-resposta à presença de algum risco (C3) mostra o resultado de aumento da aversão, indicado pela variação dos dados normalizados, concordando com os resultados encontrados por WEARY et al. (1998) e SCHÖN et al. (1999), que identificaram a resposta vocal de suínos submetidos a distintos graus de frustração e medo. 


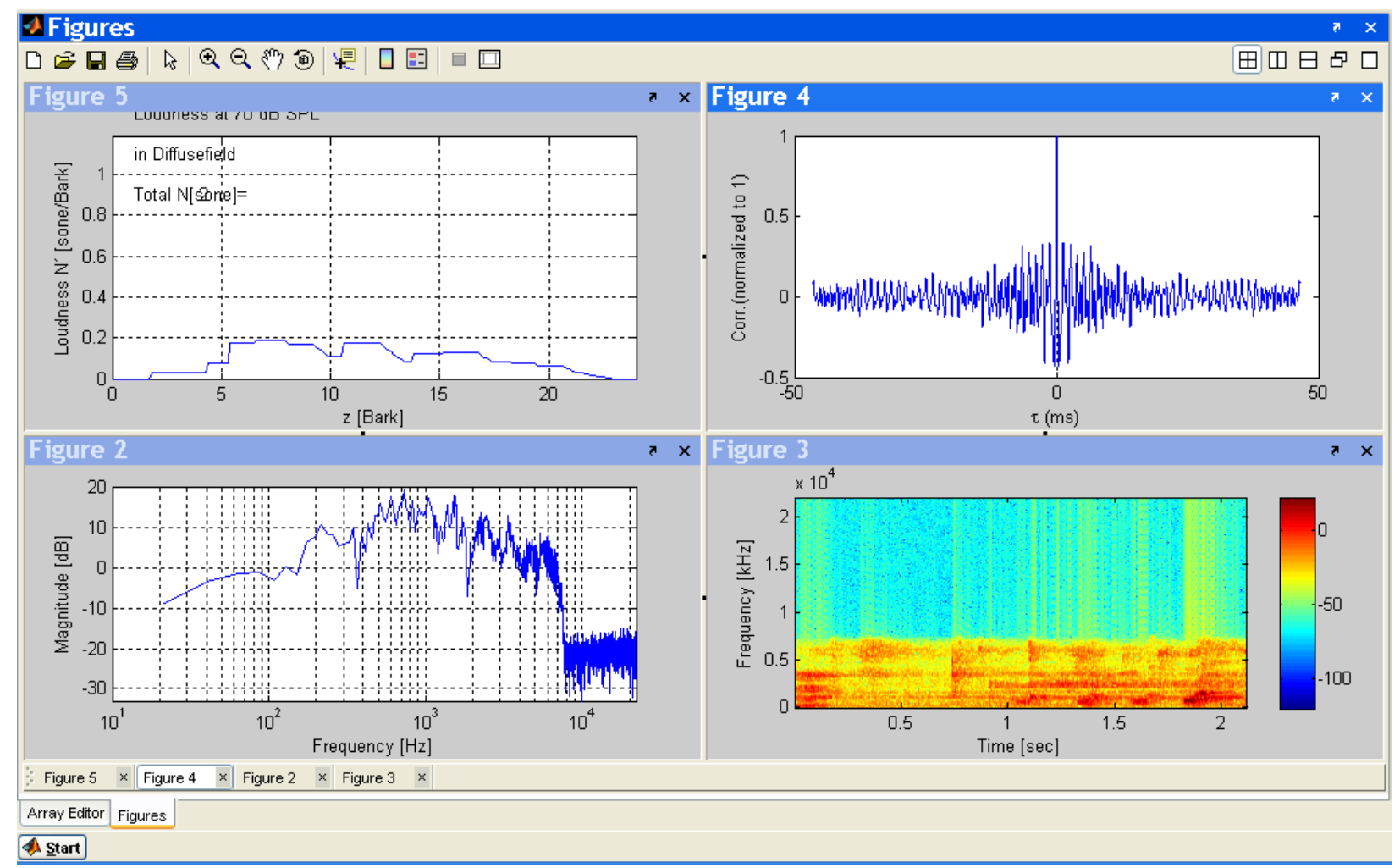

FIGURA 8. Variação da intensidade sonora, da autocorrelação, do espectro de freqüências e do espectrograma do ruído registrado de uma amostra do comportamento C1. Noise intensity variation, frequency spectrum, auto correlation and spectrogram of the registered noise of a sample from behavior $\mathrm{C} 1$.

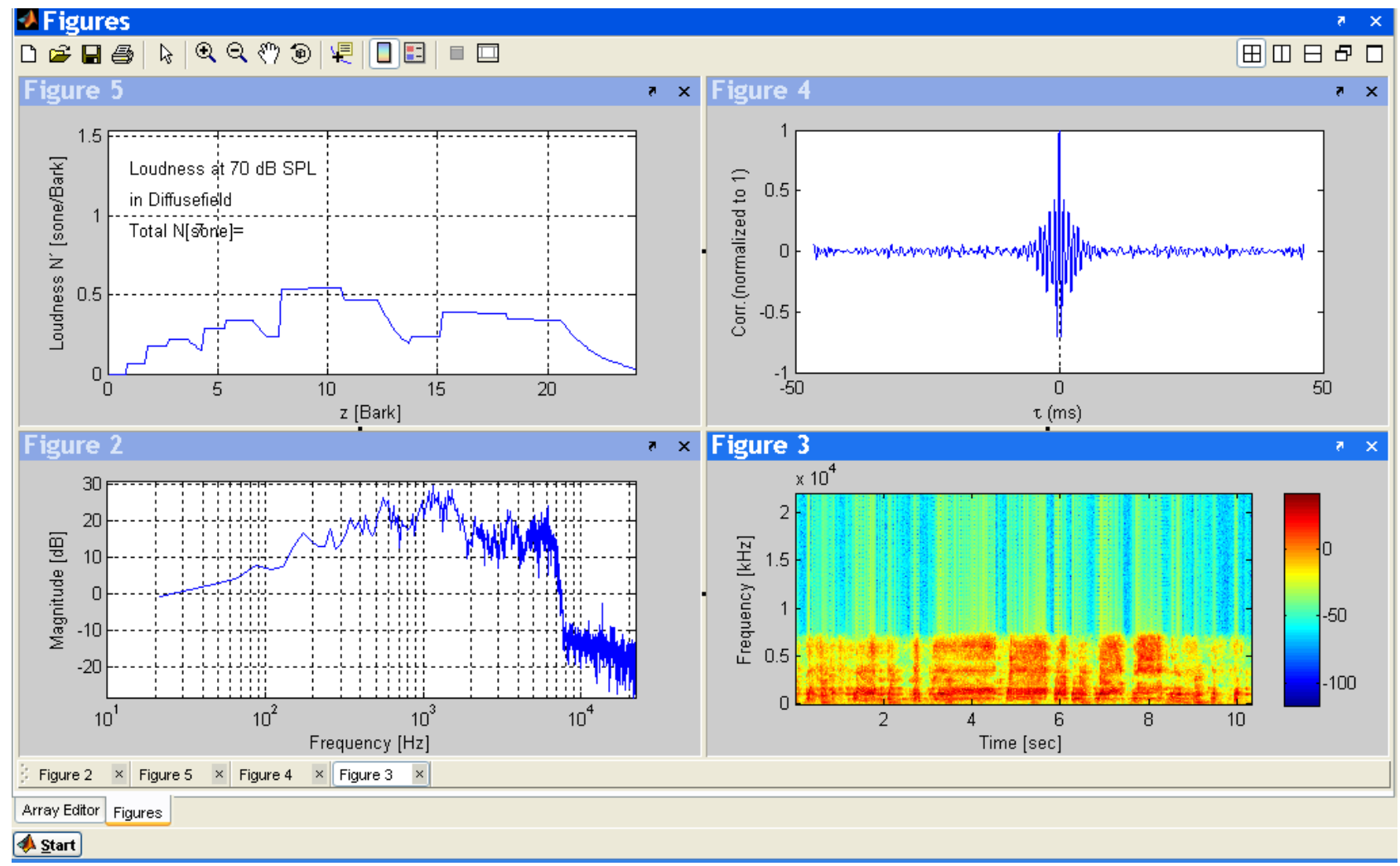

FIGURA 9. Intensidade sonora, autocorrelação, espectro de freqüências e espectrograma para o registro do ruído de uma amostra do comportamento C2. Noise intensity, auto correlation, frequency spectrum and spectrogram, for the noise sample from behavior C2. 


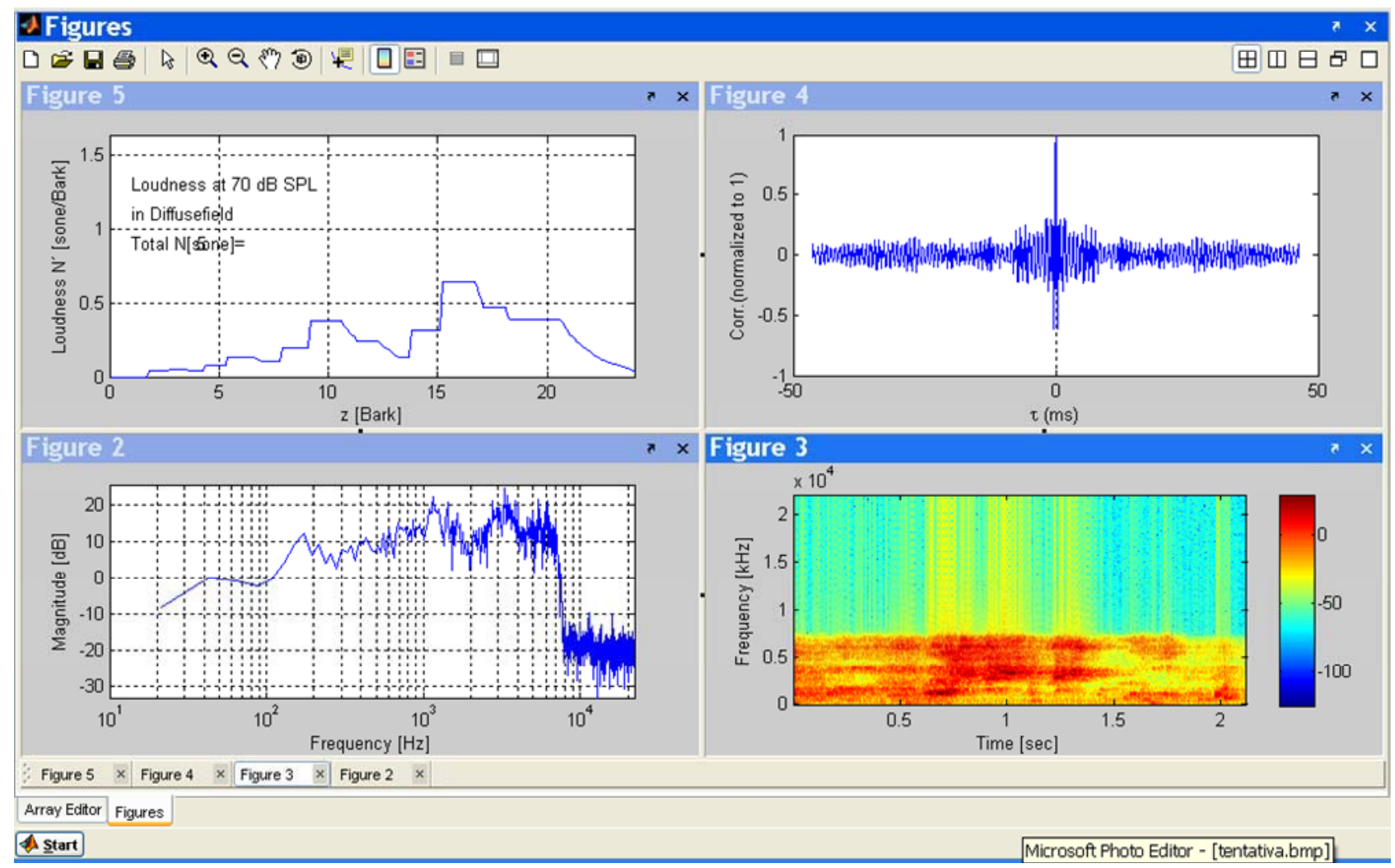

FIGURA 10. Intensidade sonora, autocorrelação, espectro de freqüências e espectrograma para o registro do ruído de uma amostra do comportamento C3. Noise intensity, auto correlation, frequency spectrum and spectrogram, for the noise sample from behavior C2.

\section{Treinamento e validação da rede}

Nas Figuras 11; 12 e 13, apresentam-se as curvas de aprendizado, considerando-se cada entrada, nas quais são indicados os índices de erro após o treinamento da rede. Nota-se que o espectro de freqüências não é um parâmetro capaz de predizer o comportamento, pois o mesmo nunca irá convergir. O parâmetro intensidade de vocalização mostrou-se eficiente na predição dos comportamentos, concordando com WEARY et al. (1998), que determinaram maior taxa de gritos agudos como indicador de dor em leitões. Também FRASER (1974) e WEARY et al. (1999) encontraram padrões de vocalização de alta intensidade sonora e repetida, acompanhada de atividade vigorosa, no período de pré-amamentação, coincidente com a presença de fome.

Os resultados acerca do desempenho da rede foram satisfatórios, e a utilização da intensidade sonora teve o melhor custo/benefício, já que são necessários poucos pontos para a sua avaliação (apenas 240). No entanto, cabe ressaltar que, quando avaliados os resultados das Figuras 11; 12 e 13, nota-se que há distinção entre os mesmos; com isso, era previsível que a rede não teria dificuldades de sucesso utilizando este algoritmo.

A análise de vocalizações de porcas na maternidade havia sido estudada por SCHÖN et al. (1999), por meio de análise multiparamétrica de sons gravados dos grunhidos da porca durante a amamentação, sendo utilizados os seguintes parâmetros: extração no domínio do tempo (em três abordagens: intervalo de ausência, número e duração dos grunhidos) e no domínio da freqüência (em três modelos: espectro simples, espectro em Quartis e Cesptrum). O parâmetro relacionado à evolução temporal do sinal desse experimento apresentou-se como característica comum entre os indivíduos, enquanto, no domínio da freqüência, traços de diferenciação individual foram encontrados pelo modelo Cesptrum, que foi obtido por meio da segunda transformação de Fourier, sendo seu objetivo o de separar as componentes periódicas das não-periódicas. 


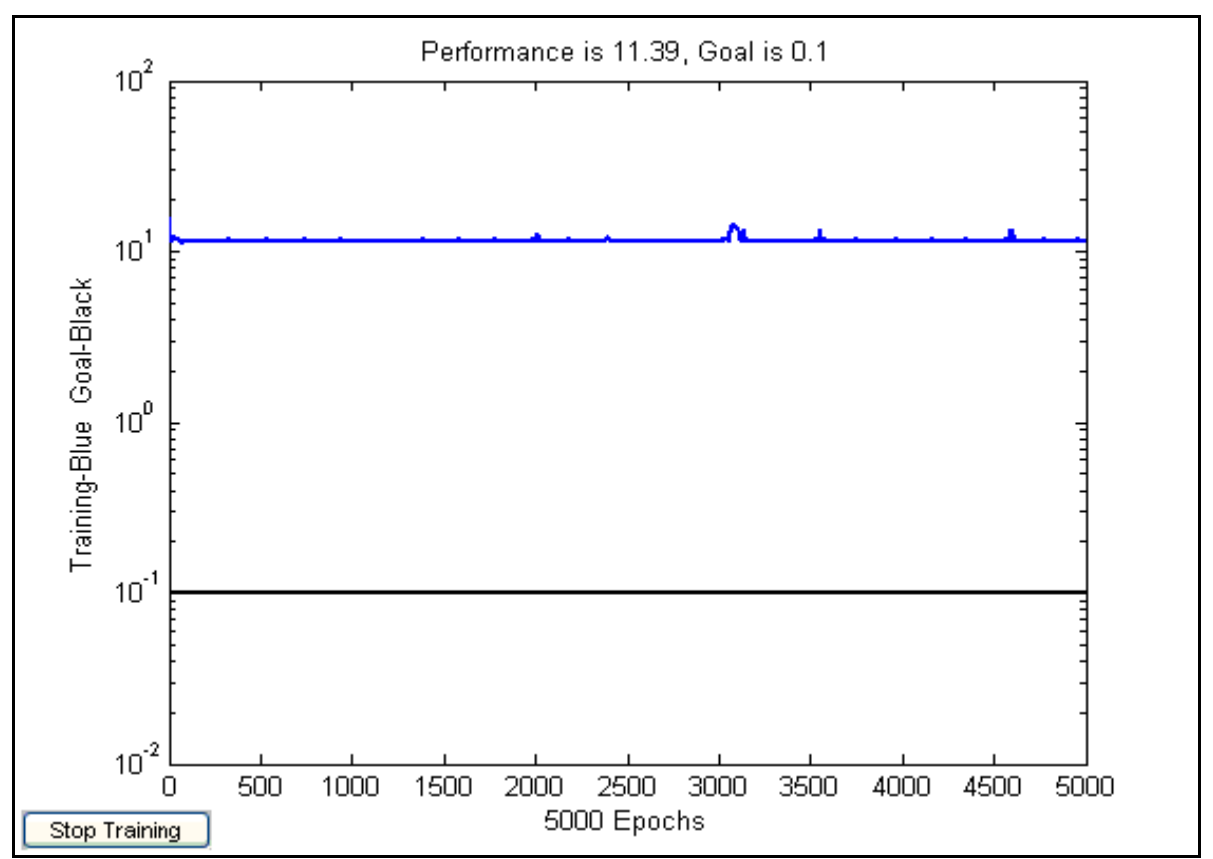

FIGURA 11. Curva de aprendizado da rede com o espectro de freqüência como parâmetro de entrada. Network learning curve with the spectrum of frequency as input parameter.

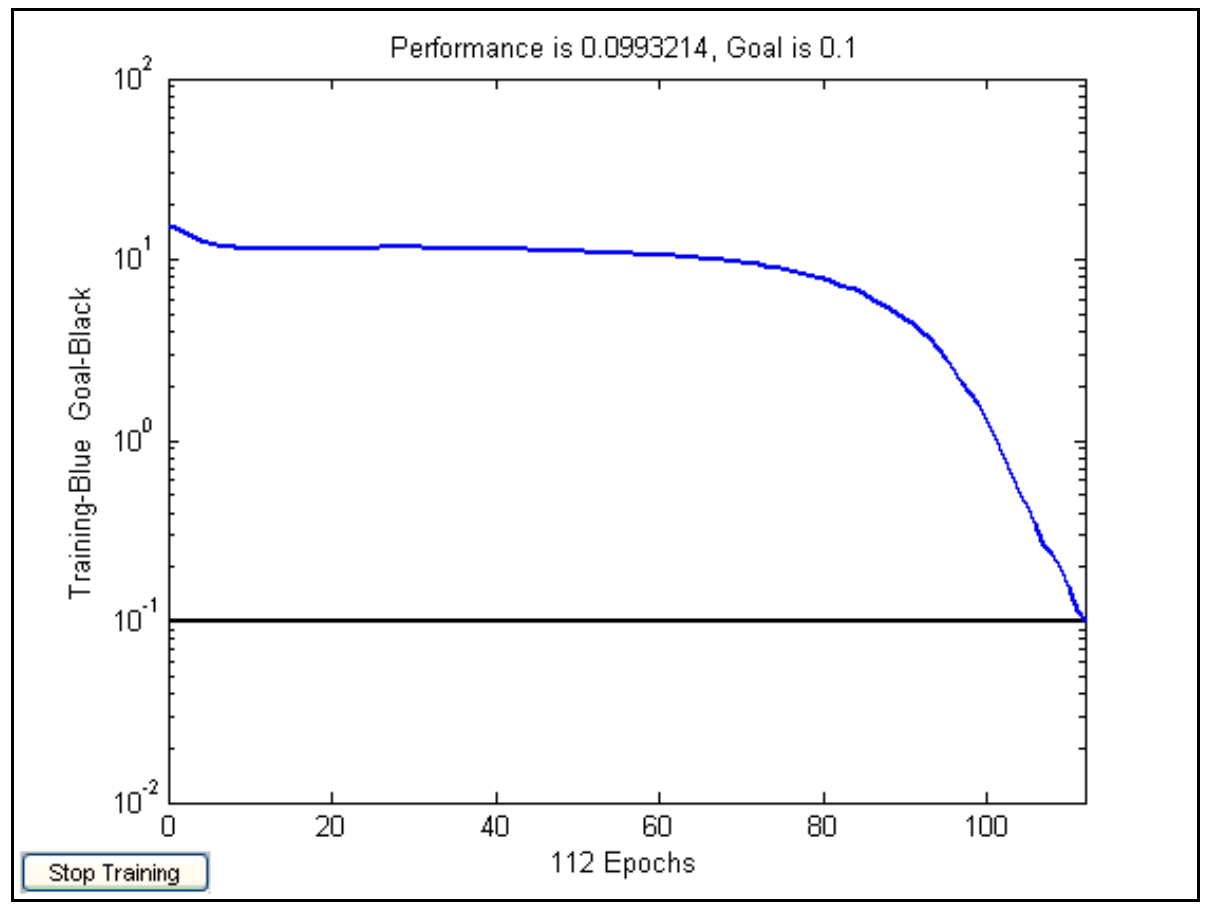

FIGURA 12. Curva de aprendizado da rede com a autocorrelação como parâmetro de entrada. Network learning curve with the auto correlation as input parameter. 


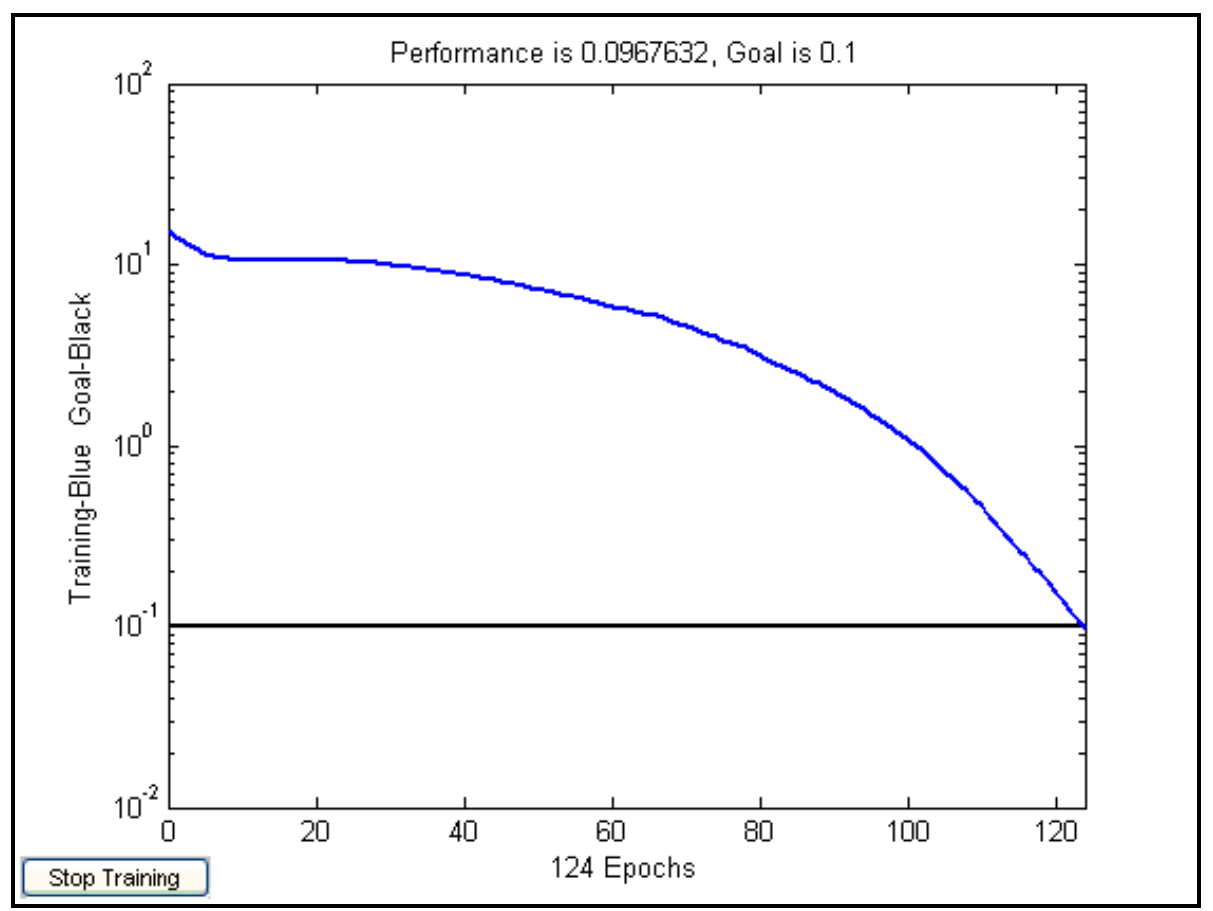

FIGURA 13. Curva de aprendizado da rede com intensidade sonora como parâmetro de entrada. Network learning curve with the noise intensity as input parameter.

Os resultados deste experimento foram similares àqueles encontrados por MANTEUFFEL \& SCHÖN (2002), uma vez que consegue reconhecer vocalizações relacionadas à presença de disputa por alimento em leitões e porcas alojados em gaiolas na maternidade.

\section{CONCLUSÕES}

Foi desenvolvido um algoritmo utilizando redes neurais artificiais, capazes de reconhecer e classificar a vocalização de leitões em fase de maternidade, relacionadas à disputa durante a amamentação e à presença de situação eventual de risco, em função da intensidade do ruído registrado.

\section{REFERÊNCIAS}

ALGERS, B.; JENSEN, P. Communication during suckling in the domestic pig effects of continuos noise. Applied Animal Behaviour Science, Londres, v.14, n.1, p.49-61, 1985.

BECKER, G.B. Bem-estar animal. 2004. Disponível em: www.porkworld.com.br. Acesso em: 9 fev. 2005.

CHEDAD, A.; MOSHOU, D.; AERTS, J.M.; HIRTUM, A. Van; RAMON, H.; BERCKMANS, D. Recognition system for pig cough based on probabilistic Neural Networks. Journal of Agricultural Research, Bristol, v.79, n.4, p.449-57, 2001.

DAWKINS, M.S.; COOK, P.A.; WHITTINGHAM; M.J.; MANSELL, K.A.; HARPER, A.E. What makes free-range broiler chickens range? In situ measurement of habitat preference. Animal Behaviour, London, v.66, n.1, p.151-60, 2003.

FRASER, A. F.; BROOM, D.M. Farm animal behaviour and welfare. $3^{\text {rd }}$ ed. Londres: B. Tindall, 1990. 246 p.

FRASER, D. The vocalization and other behaviour of growing pigs in an 'open field"' test. Applied Animal Ethology, Edinburgo, v.1, n.1, p.13-16, 1974. 
GRANDIN, T. The feasibility of using vocalization scoring as an indicator of poor welfare during slaughter. Applied Animal Behaviour Science, Bristol, v.56, n.2, p.121-8, 1998.

GRAUVOGL, A. Über das Verhalten des Hausschweines unter besonderer Berücksichtigung des Fortpflanzungsverhaltens. Berlin: Freie Universität, 1958. 112 p.

HAY, M.; VULIN, A.; GÉNIIN, S.; SALES, P.; PUNIER, A. Assessment of pain induced by castration in piglets: behavioral and physiological responses over the subsequent 5 days. Applied Animal Behavior Science, Bristol, v. 82, n.3, p.201-18, 2003.

HAYKIN, S.S. Redes neurais: princípios e práticas. Porto Alegre: Bookman, 2001. 236 p.

HEIER B.T.; HOGASEN, H.R.; JARP, J. Factors associated with mortality in Norwegian broiler flocks. Preventive Veterinary Medicine, Ithaca, v.53, n.1-2, p.147-58, 2002.

JENSEN, P.; ALGERS, B. An ethogram of piglet vocalizations during suckling. Applied Animal Ethology, Edinburgo, v.11, p.237-48, 1984.

KILEY, M. The vocalisations of ungulates, their causation and function. Tierpsychologie, Dusseldorf, v.31, n.1, p.171-222, 1972.

KLINGHOLZ, F.: MEYNHARDT, H. Lautinventare der S.augetiere - diskret oder kontinuierlich, Zeitschrift. Tierpsychologie, Dusseldorf, v.50, n.1, p.250-64, 1979.

LE NEINDRE, P.; GUÉMENÉ, D.; ARNOULD, C.; LETERRIER, C.; FAURE, J.M.; PRUNIER, A.; MEUNIER-SALAÜN, M.C. Space, environmental design and behaviour: Effect of space and environment on animal welfare. In: GLOBAL CONFERENCE ON ANIMAL WELFARE: AN OIE INITIATIVE. 2004. Disponível em: www.oie.int/eng/Welfare_2004/Conference.htm. Acesso em: 24 fev. 2005.

MANTEUFFEL, G.; PUPPE, B.; SCHÖN, P.C. Vocalization of farm animal as a measure of welfare. Applied Animal Behaviour Science, Bristol, v.88, n.1, p.163-82, 2004.

MANTEUFFEL, G.; SCHÖN, P.C. Measuring pig welfare by automatic monitoring of stress calls. Agrartechische Berichte, Bornim, v.29, n.1, p.110-18, 2002.

McINERNEY, J.P. Animal welfare, economics and policy - report on a study undertaken for the Farm \& Animal Health Economics Division of Defra, February 2004. Disponível em: www.defra.gov.uk/esg/reports/animalwelfare.pdf. Acesso em: 16 jun. 2004.

PRESCOTT, N.B.; WATHES, C.M. Preference and motivation of laying hens to eat under different illuminances and the effect of illuminance on eating behaviour. British Poultry Science, Londres, v.43, n.2, p.190-5, 2002.

REBY, D.; LEK, S.; DIMOPOULOS, I.; JOACHIM, J.; LAUGA, J.; AULAGNER, S. Artificial Neural Networks as a classification method in behavioural sciences. Behavioural Process, Londres, v.40, p.35-43, 1997.

ROLLIN, B.E. Farm animal welfare: social, bioethical, and research issues. Ames: Iowa State University Press, 1995. 168 p.

SCHÖN, P.; PUPPE, B.; GROMYKO, T.; MANTEUFFEL, G. Common features and individual differences in nurse grunting of domestic pig (Sus Scrofa) a multi-parametric analysis. Animal Behaviour, London, v.136, p.49-66, 1999.

WEARY, D.M.; APPLEBY, M.C.; FRASER, D. Response of piglets to early separation from the sow. Applied Animal Behaviour Science, Bristol, v.63, n.2, p.289-300, 1999.

WEARY, D.M.; BRAITHWAITE, L.A.; FRASER, D. Vocal response to pain in piglets. Applied Animal Behavior Science, Bristol, v.56, n.2-4, p.161-72, 1998. 
WEARY, D.M.; ROSS, S.; FRASER, D. Vocalizations by isolated piglet: reliable indicator of piglet need directed towards the sow. Applied Animal Behaviour Science, Bristol, v.53, p.249-57, 1997.

WENNRICH, G. Zum lautinventar bei haushühnern (Gallus f. domesticus). Berl Münch Tierärztl Wschr, Berlim, v.94, p.90-5, 1981. 